\title{
A novel method in determining a layered periodic structure
}

\author{
Yanli Cui ${ }^{1}$, Xiliang $\mathrm{Li}^{2}$ and Fenglong Qu ${ }^{1 *}$ (D)
}

"Correspondence:

fenglongqu@amss.ac.cn

${ }^{1}$ School of Mathematics and

Information Sciences, Yantai

University, Yantai, Shandong

264005, P.R. China

Full list of author information is

available at the end of the article

\begin{abstract}
This paper is concerned with the inverse scattering of time-harmonic waves by a penetrable structure. By applying the integral equation method, we establish the uniform $L_{\alpha}^{p}(1<p \leq 2)$ estimates for the scattered and transmitted wave fields corresponding to a series of incident point sources. Based on these a priori estimates and a mixed reciprocity relation, we prove that the penetrable structure can be uniquely identified by means of the scattered field measured only above the structure induced by a countably infinite number of quasi-periodic incident plane waves.
\end{abstract}

Keywords: Inverse scattering; Uniqueness; $L_{\alpha}^{p}$ estimate; Periodic structures

\section{Introduction}

In this paper, we consider the inverse problem of determining a penetrable periodic structure in $\mathbb{R}^{3}$ from the scattered data measured only above the structure. This kind of problem occurs in various applications such as in radar imaging, modern diffractive optics, and non-destructive testing. For convenience, we write a point $x$ in $\mathbb{R}^{3}$ for $\left(\widetilde{x}, x_{3}\right)$ with $\tilde{x}:=\left(x_{1}, x_{2}\right) \in \mathbb{R}^{2}$. Assume that the penetrable profile is described by

$$
\Gamma:=\left\{x \in \mathbb{R}^{3}: x_{3}=f(\widetilde{x})\right\},
$$

where $f$ is a periodic function with respect to the variable $\widetilde{x}$, that is, $f(\widetilde{x})=f(\widetilde{x}+2 n \pi)$ for $n:=\left(n_{1}, n_{2}\right) \in \mathbb{Z}^{2}$. Assume further that the homogeneous media above and below $\Gamma$ are described by

$$
\Omega_{+}:=\left\{x \in \mathbb{R}^{3}: x_{3}>f(\widetilde{x})\right\} \quad \text { and } \quad \Omega_{-}:=\left\{x \in \mathbb{R}^{3}: x_{3}<f(\widetilde{x})\right\}
$$

with the wave numbers $k_{1}$ and $k_{2}$, respectively.

Consider the incident plane waves in the form of

$$
u^{i}(x)=\exp \left(i \alpha_{j} \cdot \tilde{x}-i \beta_{j}^{+} x_{3}\right), \quad j \in \mathbb{Z}^{2}, \text { with } \alpha_{j}=\alpha+j,
$$

(c) The Author(s) 2020. This article is licensed under a Creative Commons Attribution 4.0 International License, which permits use, sharing, adaptation, distribution and reproduction in any medium or format, as long as you give appropriate credit to the original author(s) and the source, provide a link to the Creative Commons licence, and indicate if changes were made. The images or other third party material in this article are included in the article's Creative Commons licence, unless indicated otherwise in a credit line to the material. If material is not included in the article's Creative Commons licence and your intended use is not permitted by statutory regulation or exceeds the permitted use, you will need to obtain permission directly from the copyright holder. To view a copy of this licence, visit http://creativecommons.org/licenses/by/4.0/. 
which propagate downward from $\Omega_{+}$with $\alpha=\left(\alpha_{1}, \alpha_{2}\right):=k_{1}\left(\sin \theta_{1} \cos \theta_{2}, \sin \theta_{1} \sin \theta_{2}\right)$ with the incident angle $\theta_{1} \in[0, \pi / 2), \theta_{2} \in[0,2 \pi)$, and $\beta_{j}^{+} \in \mathbb{C}$ is given by

$$
\beta_{j}^{+}=\sqrt{k_{1}^{2}-\left|\alpha_{j}\right|^{2}} \quad \text { if }\left|\alpha_{j}\right| \leq k_{1}, \quad \beta_{j}^{+}=i \sqrt{\left|\alpha_{j}\right|^{2}-k_{1}^{2}} \quad \text { if }\left|\alpha_{j}\right|>k_{1} .
$$

Then the scattering of the incident $u^{i}$ by the periodic structure can be formulated in determining the total field $u_{1}:=u^{i}+u^{s}$ with the scattered field $u^{s}$ and the transmitted field $u_{2}$ to the following problem:

$$
\begin{aligned}
& \triangle u_{1}+k_{1}^{2} u_{1}=0 \quad \text { in } \Omega_{+}, \\
& \triangle u_{2}+k_{2}^{2} u_{2}=0 \quad \text { in } \Omega_{-}, \\
& u_{1}=u_{2}, \quad \frac{\partial u_{1}}{\partial v}=\lambda \frac{\partial u_{2}}{\partial v} \quad \text { on } \Gamma, \\
& u^{s}(x)=\sum_{n \in \mathbb{Z}^{2}} u_{n}^{+} \exp \left(i \alpha_{n} \cdot \tilde{x}+i \beta_{n}^{+} x_{3}\right), \quad x_{3}>A_{1}:=\max _{t \in \mathbb{R}^{2}} f(t), \\
& u_{2}(x)=\sum_{n \in \mathbb{Z}^{2}} u_{n}^{-} \exp \left(i \alpha_{n} \cdot \tilde{x}-i \beta_{n}^{-} x_{3}\right), \quad x_{3}<A_{2}:=\min _{t \in \mathbb{R}^{2}} f(t) .
\end{aligned}
$$

Here, $u_{n}^{ \pm} \in \mathbb{C}$ are the solution sequences, $\lambda$ is the transmission coefficient and the unit normal vector $v$ on $\Gamma$ is directed into the interior of $\Omega_{-}$. Notice that the incident wave $u^{i}(\cdot)$ satisfies such an $\alpha$-quasi-periodic condition $u^{i}\left(\widetilde{x}+2 n \pi, x_{3}\right)=e^{i 2 \alpha \cdot n \pi} u^{i}\left(\widetilde{x}, x_{3}\right)$ for all $n \in \mathbb{Z}^{2}$. Then the solution $u_{l}, l=1,2$, is also required to satisfy the same $\alpha$-quasi-periodic condition, i.e., $u_{l}\left(\widetilde{x}+2 n \pi, x_{3}\right)=e^{i 2 \alpha \cdot n \pi} u_{l}\left(\widetilde{x}, x_{3}\right)$ in $\mathbb{R}^{3}$. Conditions (1.5) and (1.6) are known as the Rayleigh expansion conditions of the scattered field $u^{s}$ in $\Omega_{+}$and the transmitted field $u_{2}$ in $\Omega_{-}$, respectively, with $\beta_{n}^{-}$defined similarly as $\beta_{n}^{+}$by the wave number $k_{2}$.

The well-posedness of problem (1.2)-(1.6) can be established by the variational method (cf. [31]) or the integral equation method (cf. [32, 33]). In the current paper we first establish the $L_{\alpha}^{p}(1<p \leq 2)$ estimates for the scattered field $u^{s}$ and the transmitted field $u_{2}$. Based on these a priori estimates, we focus on the unique identification of the penetrable periodic structure from the scattered field $u^{s}$ measured only on a straight line above the periodic structure induced by a countably infinite number of quasi-periodic incident plane waves.

There are lots of results concerning the uniqueness issue for the inverse periodic transmission problems (cf. $[5,7,12,13,18,19,23,24,33,34])$ and for the inverse scattering by the polygonal periodic structure (cf. $[6,11,14])$. For the special case when the medium has the energy absorption property, a uniqueness theorem was obtained in [5] from the measured scattered field for one incident plane wave in a two-dimensional space. The result of [5] was then extended to the three-dimensional case in [2]. It should be remarked that the uniqueness with one incident wave does not hold true for the inverse periodic problem for a real wave number case, that is, the medium does not has a property of energy absorption. See also [7] for a uniqueness theorem on the recovery of a smooth periodic structure with one incident plane wave under some a priori assumptions on the structure. For the case when a priori restrictions on the height of the grating surface are known in advance, a uniqueness result can be found in [18] on the identification of a smooth perfectly reflecting periodic structure from many measurements corresponding to a finite number of incident 
plane waves. The method of [18] was extended to the periodic transmission problem [13]. There also exist some numerical methods in reconstructing periodic structures. For example, a linear sampling method was developed in [20,22] for determining the shape of partially coated bi-periodic structures, and in [35] a novel linear sampling method was introduced for simultaneously reconstructing dielectric grating structures in an inhomogeneous periodic medium. See also [10] for a finite element method or $[3,4,17]$ for the factorization method in determining the periodic structures, or [30] for the uniquely reconstruction of a locally perturbed infinite plane. Recently, by making use of the differential sampling method, the anisotropic periodic layer can be uniquely determined in [25] under the assumption that the complement of the periodic layer in one period is connected. The analysis of sampling methods for the recovery of a local perturbation in a periodic layer can be found in [16].

For the scattering by general periodic structures case, there are several uniqueness results. We refer to [23] for a uniqueness theorem for the inverse Dirichlet problem, and to $[21,24,32]$ for uniqueness results for the inverse transmission problem by means of all quasi-periodic incident plane waves. The reader is referred to [19] for a partially coated perfectly grating case with respect to infinitely many point sources, and to [34] for uniqueness results for both the partially coated perfectly reflecting grating and the periodic transmission case in a two-dimensional space, corresponding to a countably infinite number of quasi-periodic incident plane waves. In this paper we intend to develop a novel method, which differs from the approach used in [34], to prove the uniqueness on the identification of the penetrable periodic structure in the three-dimensional space from the measured data only above the structure with respect to a countably infinite number of quasiperiodic incident plane waves. The technique developed in this paper can date back to the work $[27,36]$ on the inverse scattering problems of determining the support of penetrable electromagnetic obstacles or to [28] for the fluid-solid interaction problem of identifying the bounded solid obstacle, [29] for the cavity scattering case.

The paper is organized as follows. In Sect. 2, the a priori estimates in the sense of $L_{\alpha}^{p}(1<p \leq 2)$ norm for the solution of the direct scattering problem in $\mathbb{R}^{3}$ are established by applying the integral equation method. Section 3 is devoted to the inverse problem of uniquely determining the periodic structure from the measured data only above the structure produced by a countably infinite number of quasi-periodic incident plane waves.

\section{A priori estimates}

In this section we establish some a priori estimates for the solution of the direct scattering problem by employing the integral equation method. Eliminating the incident field $u^{i}$, it is easily found that the scattered field $w_{1}:=u_{1}-u^{i}$ in $\Omega_{+}$and the transmitted field $w_{2}:=u_{2}$ in $\Omega_{-}$satisfy the following boundary value problem:

$$
\begin{array}{ll}
\triangle w_{1}+k_{1}^{2} w_{1}=0 & \text { in } \Omega_{+}, \\
\triangle w_{2}+k_{2}^{2} w_{2}=0 & \text { in } \Omega_{-}, \\
w_{1}-w_{2}=f_{1}, \quad \frac{\partial w_{1}}{\partial v}-\lambda \frac{\partial w_{2}}{\partial v}=f_{2} & \text { on } \Gamma, \\
w_{1}(x)=\sum_{n \in \mathbb{Z}^{2}} w_{n}^{+} \exp \left(i \alpha_{n} \cdot \tilde{x}+i \beta_{n}^{+} x_{3}\right), & x_{3}>A_{1},
\end{array}
$$




$$
w_{2}(x)=\sum_{n \in \mathbb{Z}^{2}} w_{n}^{-} \exp \left(i \alpha_{n} \cdot \tilde{x}-i \beta_{n}^{-} x_{3}\right), \quad x_{3}<A_{2}
$$

in the general case $f_{1}, f_{2} \in L_{\alpha}^{p}(\Gamma)$ with $1<p \leq 2$. Here, $L_{\alpha}^{p}(\Gamma)(p \geq 1)$ denotes the Sobolev space of scalar functions on $\Gamma$ which is assumed to be $\alpha$-quasi-periodic with respect to the variable $\tilde{x}$, equipped with the norm in the usual Sobolev space $L^{p}(\Gamma)$.

Before going further we first introduce the basic notations that are used in the rest of this paper. For simplicity, we use $\Omega_{ \pm}$and $\Gamma$ again to denote the same sets restricted to one period $0<x_{1}, x_{2}<2 \pi$. For each $h>0$, denote by $\Omega_{+}(h):=\left\{x \in \Omega_{+}: x_{3}<A_{1}+h\right\}, \Omega_{-}(h):=$ $\left\{x \in \Omega_{-}: x_{3}>A_{2}-h\right\}, \Gamma_{+}(h):=\left\{x \in \Omega_{+}: x_{3}=A_{1}+h\right\}$, and $\Gamma_{-}(h):=\left\{x \in \Omega_{-}: x_{3}=A_{2}-\right.$ $h\}$, respectively. Then, let $H_{\alpha}^{1}\left(\Omega_{ \pm}(h)\right)$ and $L_{\alpha}^{p}\left(\Omega_{ \pm}(h)\right)(p \geq 1)$ denote the Sobolev spaces of scalar functions on $\Omega_{ \pm}(h)$ which are assumed to be $\alpha$-quasi-periodic with respect to the variable $\tilde{x}$, equipped with the norms in the usual Sobolev spaces $H^{1}\left(\Omega_{ \pm}(h)\right)$ and $L^{p}\left(\Omega_{ \pm}(h)\right)$, respectively. Let $H_{\alpha}^{1 / 2}\left(\Gamma_{ \pm}(h)\right)$ denote the trace space of $H_{\alpha}^{1}\left(\Omega_{ \pm}(h)\right)$, and $H_{\alpha}^{-1 / 2}\left(\Gamma_{ \pm}(h)\right)$ is the dual space of $H_{\alpha}^{1 / 2}\left(\Gamma_{ \pm}(h)\right)$.

We introduce the free space $\alpha$-quasi-periodic Green function

$$
G_{1}\left(x, y ; k_{1}\right)=\frac{i}{8 \pi^{2}} \sum_{n \in \mathbb{Z}^{2}} \frac{1}{\beta_{n}^{+}} \exp \left(i \alpha_{n} \cdot(\tilde{x}-\widetilde{y})+i \beta_{n}^{+}\left|x_{3}-y_{3}\right|\right), \quad x \neq y
$$

and the $\alpha$-quasi-periodic layer-potential operators $S_{1}, K_{1}, K_{1}^{\prime}$, and $T_{1}$ defined by

$$
\begin{aligned}
& S_{1} \xi(x)=\int_{\Gamma} G_{1}\left(x, y ; k_{1}\right) \xi(y) d s(y), \quad x \in \Gamma, \\
& K_{1} \xi(x)=\int_{\Gamma} \frac{\partial}{\partial v(y)} G_{1}\left(x, y ; k_{1}\right) \xi(y) d s(y), \quad x \in \Gamma, \\
& K_{1}^{\prime} \xi(x)=\frac{\partial}{\partial \nu(x)} \int_{\Gamma} G_{1}\left(x, y ; k_{1}\right) \xi(y) d s(y), \quad x \in \Gamma, \\
& T_{1} \xi(x)=-\frac{\partial}{\partial \nu(x)} \int_{\Gamma} \frac{\partial}{\partial v(y)} G_{1}\left(x, y ; k_{1}\right) \xi(y) d s(y), \quad x \in \Gamma .
\end{aligned}
$$

Noting that $G_{1}\left(x, y ; k_{1}\right)-\Phi\left(x, y ; k_{1}\right)$ is smooth, it follows from [8] that the operators $S_{1}: H_{\alpha}^{-\frac{1}{2}}(\Gamma) \rightarrow H_{\alpha}^{\frac{1}{2}}(\Gamma), K_{1}: H_{\alpha}^{\frac{1}{2}}(\Gamma) \rightarrow H_{\alpha}^{\frac{1}{2}}(\Gamma), K_{j}^{\prime}: H_{\alpha}^{-\frac{1}{2}}(\Gamma) \rightarrow H_{\alpha}^{-\frac{1}{2}}(\Gamma)$, and $T_{1}: H_{\alpha}^{\frac{1}{2}}(\Gamma) \rightarrow$ $H_{\alpha}^{-\frac{1}{2}}(\Gamma)$ are all bounded, where $\Phi\left(x, y ; k_{1}\right)=\frac{1}{4 \pi} \frac{e^{i k_{1}|x-y|}}{|x-y|}$ is the fundamental solution of the Helmholtz equation $\triangle \Phi+k_{1}^{2} \Phi=-\delta_{y}$ in the free space $\mathbb{R}^{3}$.

Theorem 2.1 For $f_{1}, f_{2} \in L_{\alpha}^{p}(\Gamma)$ with $1<p \leq 2$, there exists a unique solution $\left(w_{1}, w_{2}\right) \in$ $L_{\alpha}^{p}\left(\Omega_{+}(h)\right) \times L_{\alpha}^{p}\left(\Omega_{-}(h)\right)$ to the transmission problem $(2.1)-(2.5)$ satisfying the estimate

$$
\left\|w_{1}\right\|_{L_{\alpha}^{p}\left(\Omega_{+}(h)\right)}+\left\|w_{2}\right\|_{L_{\alpha}^{p}\left(\Omega_{-}(h)\right)} \leq C\left(\left\|f_{1}\right\|_{L_{\alpha}^{p}(\Gamma)}+\left\|f_{2}\right\|_{L_{\alpha}^{p}(\Gamma)}\right),
$$

where $C>0$ is a constant independent of $f_{1}, f_{2}$, and depending on $G_{j}\left(\cdot, y ; k_{j}\right), \Omega_{+}(h)$ with $j=1,2$ and the boundedness of the operators $S_{j}, K_{j}, K_{j}^{\prime}, j=1,2$, and $T_{2}-T_{1}$ in $L_{\alpha}^{p}(\Gamma)$.

Moreover, if $f_{1}, f_{2} \in L_{\alpha}^{p}(\Gamma)$ with $\frac{4}{3}<p \leq 2$, we have

$$
\left\|w_{1}\right\|_{L_{\alpha}^{2}\left(\Omega_{+}(h)\right)}+\left\|w_{2}\right\|_{L_{\alpha}^{2}\left(\Omega_{-}(h)\right)} \leq C\left(\left\|f_{1}\right\|_{L_{\alpha}^{p}(\Gamma)}+\left\|f_{2}\right\|_{L_{\alpha}^{p}(\Gamma)}\right)
$$


with a positive constant $C>0$, which is independent of $f_{1}, f_{2}$, and depending on $G_{j}\left(\cdot, y ; k_{j}\right)$, $\Omega_{+}(h)$ with $j=1,2$ and the boundedness of the operators $S_{j}, K_{j}, K_{j}^{\prime}, j=1,2$ and $T_{2}-T_{1}$ in $L_{\alpha}^{p}(\Gamma)$.

Proof We seek a solution of problem (2.1)-(2.5) in the form of combined single- and double-layer potential

$$
\begin{aligned}
& w_{1}(x)=\int_{\Gamma} G_{1}\left(x, y ; k_{1}\right) \varphi_{1}(y) d s(y)+\lambda \int_{\Gamma} \frac{\partial G_{1}\left(x, y ; k_{1}\right)}{\partial v(y)} \varphi_{2}(y) d s(y), \\
& w_{2}(x)=\int_{\Gamma} G_{2}\left(x, y ; k_{2}\right) \varphi_{1}(y) d s(y)+\int_{\Gamma} \frac{\partial G_{2}\left(x, y ; k_{2}\right)}{\partial v(y)} \varphi_{2}(y) d s(y),
\end{aligned}
$$

where $G_{2}\left(x, y ; k_{2}\right)$ is defined as (2.6) with the wave number $k_{1}$ replaced by $k_{2}$.

With the aid of the jump relations of the layer potentials (see [26] for the case in the $L^{p}$ norm), we obtain that the transmission problem (2.1)-(2.5) can be reduced to the system of integral equations

$$
\left(\begin{array}{l}
\varphi_{2} \\
\varphi_{1}
\end{array}\right)+L\left(\begin{array}{c}
\varphi_{2} \\
\varphi_{1}
\end{array}\right)=\left(\begin{array}{c}
\frac{2}{1+\lambda} f_{1} \\
-\frac{2}{1+\lambda} f_{2}
\end{array}\right) \quad \text { in } L_{\alpha}^{p}(\Gamma) \times L_{\alpha}^{p}(\Gamma),
$$

where the operator $L$ is given by

$$
L:=\left(\begin{array}{cc}
\frac{2}{1+\lambda}\left(\lambda K_{1}-K_{2}\right) & \frac{2}{1+\lambda}\left(S_{1}-S_{2}\right) \\
\frac{2 \lambda}{1+\lambda}\left(T_{2}-T_{1}\right) & \frac{2}{1+\lambda}\left(\lambda K_{2}^{\prime}-K_{1}^{\prime}\right)
\end{array}\right) .
$$

It is easily shown that (2.15) is of Fredholm type due to the compactness of the operators $S_{j}, K_{j}, K_{j}^{\prime}, j=1,2$, and $T_{2}-T_{1}$ in $L_{\alpha}^{p}(\Gamma)$. This, together with the uniqueness of the scattering problem (1.2)-(1.6), implies that (2.15) has a unique solution $\left(\varphi_{2}, \varphi_{1}\right)^{T} \in L_{\alpha}^{p}(\Gamma) \times L_{\alpha}^{p}(\Gamma)$ with the estimate

$$
\left\|\varphi_{2}\right\|_{L_{\alpha}^{p}(\Gamma)}+\left\|\varphi_{1}\right\|_{L_{\alpha}^{p}(\Gamma)} \leq C\left(\left\|f_{1}\right\|_{L_{\alpha}^{p}(\Gamma)}+\left\|f_{2}\right\|_{L_{\alpha}^{p}(\Gamma)}\right)
$$

We next prove the $L_{\alpha}^{p}, 1<p \leq 2$ estimates for the solution of the transmission problem (2.1)-(2.5). In fact, it can be checked that

$$
\begin{aligned}
& \left\|\int_{\Gamma} \Omega_{+}(h) G_{1}\left(\cdot, y ; k_{1}\right) \varphi_{1}(y) d s(y)\right\|_{L_{\alpha}^{p}\left(\Omega_{+}(h)\right)} \\
& =\sup _{g \in L_{\alpha}^{q},\|g\|_{L_{\alpha}^{q}\left(\Omega_{+}(h)\right)}}\left|\int_{\Omega_{+}(h)} \int_{\Gamma} G_{1}\left(x, y ; k_{1}\right) \varphi_{1}(y) d s(y) g(x) d x\right| \\
& =\sup _{g \in L_{\alpha}^{q},\|g\|_{L_{\alpha}^{q}\left(\Omega_{+}(h)\right)}}\left|\int_{\Gamma} \int_{\Omega_{+}(h)} G_{1}\left(x, y ; k_{1}\right) g(x) d x \varphi_{1}(y) d s(y)\right| \\
& \quad \leq|\Gamma|^{\frac{1}{q}} \sup _{g \in L^{q},\|g\|_{L_{\alpha}^{q}\left(\Omega_{+}(h)\right)}=1} \sup _{y \in \Gamma}\left\|G_{1}\left(\cdot, y ; k_{1}\right)\right\|_{L_{\alpha}^{p}\left(\Omega_{+}(h)\right)}\|g\|_{L_{\alpha}^{q}\left(\Omega_{+}(h)\right)}\left\|\varphi_{1}\right\|_{L_{\alpha}^{p}(\Gamma)} \\
& \quad=|\Gamma|^{\frac{1}{q}} \sup _{y \in \Gamma}\left\|G_{1}\left(\cdot, y ; k_{1}\right)\right\|_{L_{\alpha}^{p}\left(\Omega_{+}(h)\right)}\left\|\varphi_{1}\right\|_{L_{\alpha}^{p}(\Gamma)} \leq C\left\|\varphi_{1}\right\|_{L_{\alpha}^{p}(\Gamma)}
\end{aligned}
$$


and

$$
\begin{aligned}
& \left\|\int_{\Gamma} \frac{\partial G_{1}\left(\cdot, y ; k_{1}\right)}{\partial v(y)} \varphi_{2}(y) d s(y)\right\|_{L_{\alpha}^{p}\left(\Omega_{+}(h)\right)} \\
& =\sup _{g \in L_{\alpha}^{q},\|g\|_{L_{\alpha}^{q}\left(\Omega_{+}(h)\right)}=1}\left|\int_{\Omega_{+}(h)} \int_{\Gamma} \frac{\partial G_{1}\left(x, y ; k_{1}\right)}{\partial \nu(y)} \varphi_{2}(y) d s(y) g(x) d x\right| \\
& =\sup _{g \in L_{\alpha}^{q},\|g\|_{L_{\alpha}^{q}\left(\Omega_{+}(h)\right)}=1}\left|\int_{\Gamma} \frac{\partial}{\partial v(y)} \int_{\Omega_{+}(h)} G_{1}\left(x, y ; k_{1}\right) g(x) d x \varphi_{2}(y) d s(y)\right| \\
& \leq \sup _{g \in L_{\alpha}^{q},\|g\|_{L_{\alpha}^{q}\left(\Omega_{+}(h)\right)}=1}\left\|\frac{\partial}{\partial v(y)} \int_{\Omega_{+}(h)} G_{1}\left(x, \cdot ; k_{1}\right) g(x) d x\right\|_{L_{\alpha}^{q}(\Gamma)}\left\|\varphi_{2}\right\|_{L_{\alpha}^{p}(\Gamma)} \\
& \leq \sup _{g \in L_{\alpha}^{q},\|g\|_{L_{\alpha}^{q}\left(\Omega_{+}(h)\right)}=1} C\|g\|_{L_{\alpha}^{q}\left(\Omega_{+}(h)\right)} \cdot\left\|\varphi_{2}\right\|_{L_{\alpha}^{p}(\Gamma)}=C\left\|\varphi_{2}\right\|_{L_{\alpha}^{p}(\Gamma)}
\end{aligned}
$$

with $\frac{1}{p}+\frac{1}{q}=1$. Here, we have used the fact that the volume potential operator is bounded from $L_{\alpha}^{q}\left(\Omega_{+}(h)\right)$ into $W_{\alpha}^{2, q}\left(\Omega_{+}(h)\right)$ with $2 \leq q \leq 4$ (see [15, Theorem 9.9]), and the boundary trace operator is bounded from $W_{\alpha}^{1, q}\left(\Omega_{+}(h)\right)$ into $L_{\alpha}^{q}(\Gamma)$ with $2 \leq q \leq 4$ (see [1, Theorem 5.36]). It is noted that (2.17)-(2.18) still holds true, with $G_{1}\left(x, \cdot ; k_{1}\right)$ replaced by $G_{2}\left(x, \cdot ; k_{2}\right)$ and $\Omega_{+}(h)$ replaced by $\Omega_{-}(h)$, respectively. Now the desired estimate $(2.11)$ follows from (2.13)-(2.14) and (2.16)-(2.18). Furthermore, if $f_{1}, f_{2} \in L_{\alpha}^{p}(\Gamma)$ with $\frac{4}{3}<p \leq 2$, by the similar arguments as those in (2.17)-(2.18), one can derive the required result (2.13). This completes the proof of the theorem.

Corollary 2.2 For $y_{0} \in \Gamma$, define the sequence $y_{j}:=y_{0}-\frac{1}{j} v\left(y_{0}\right) \in \Omega_{+}, j \in \mathbb{N}$. Let $\left(u_{1 j}, u_{2 j}\right)$ be the solution of the scattering problem (1.2)-(1.6) with the incident point source $u^{i}=$ $G_{1}\left(x, y_{j} ; k_{1}\right)$. Then, for any $h \in \mathbb{R}$, we have

$$
\left\|u_{1 j}\right\|_{L_{\alpha}^{2}\left(\Omega_{+}(h)\right)}+\left\|u_{2 j}\right\|_{L_{\alpha}^{2}\left(\Omega_{-}(h)\right)} \leq C
$$

uniformly for $j \in \mathbb{N}_{+}$, where $C>0$ is a constant depending on $G_{j}\left(\cdot, y ; k_{j}\right), \Omega_{+}(h)$ with $j=1,2$.

Proof It is obvious that $\left(u_{1 j}^{s}, u_{2 j}\right)$ satisfies problem (2.1)-(2.5) with the boundary data

$$
f_{1}(j):=-G_{1}\left(x, y_{j} ; k_{1}\right), \quad f_{2}(j):=-\frac{\partial G_{1}\left(x, y_{j} ; k_{1}\right)}{\partial v} \quad j \in \mathbb{N}
$$

It is easy to see that $f_{1}(j), f_{2}(j) \in L_{\alpha}^{p}(\Gamma)$ are uniformly bounded for $j \in \mathbb{N}$ with $\frac{4}{3}<p<\frac{3}{2}$. Then the required result (2.19) follows from Theorem 2.1. This proves the corollary.

Theorem 2.3 Let $\left(u_{1 j}, u_{2 j}\right)$ be the solution of the scattering problem (1.2)-(1.6) corresponding to the incident point source $u^{i}=G_{1}\left(x, y_{j} ; k_{1}\right)$ with $y_{j}$ defined in Corollary 2.2. Then, for any $h \in \mathbb{R}$, it holds that

$$
\left\|u_{2 j}\right\|_{H_{\alpha}^{1}\left(\Omega_{-}(h) \backslash \bar{B}\right)} \leq C
$$


uniformly for $j \in \mathbb{N}_{+}$. Here, $C>0$ is a constant depending on $G_{j}\left(\cdot, y ; k_{j}\right), \Omega_{+}(h)$ with $j=1,2$ and the uniform boundedness of $S_{\Gamma \backslash B}(j)$ and $K_{\Gamma \backslash B}(j)$ in the corresponding Hilbert spaces, $B$ is a ball satisfying that $B \supset B_{\delta}$, and $B_{\delta}$ is a small ball centered at $y_{0}$ with the radius $\delta>0$.

Proof Define $\tilde{y}_{j}:=y_{0}+\frac{1}{j} v\left(y_{0}\right) \in \Omega_{-}$, let $w_{1}(j):=u_{1 j}^{s}-G_{1}\left(x, \tilde{y}_{j} ; k_{1}\right)$ and $w_{2}(j):=u_{2 j}$, it follows that $\left(w_{1}(j), w_{2}(j)\right)$ satisfies problem $(2.1)-(2.5)$ with the boundary data

$$
\begin{aligned}
& f_{1}(j):=-G_{1}\left(x, y_{j} ; k_{1}\right)-G_{1}\left(x, \tilde{y}_{j} ; k_{1}\right), \\
& f_{2}(j):=-\frac{\partial G_{1}\left(x, y_{j} ; k_{1}\right)}{\partial v}-\frac{\partial G_{1}\left(x, \tilde{y}_{j} ; k_{1}\right)}{\partial v} .
\end{aligned}
$$

Obviously, $f_{1}(j) \in L_{\alpha}^{p}(\Gamma)$ is uniformly bounded for $j \in \mathbb{N}$, where $1<p<2$. Furthermore, it is seen from [9, Lemma 4.2] that $f_{2}(j) \in C(\Gamma)$ is uniformly bounded for $j \in \mathbb{N}$. So $f_{2}(j) \in L_{\alpha}^{p}(\Gamma)$ is uniformly bounded for $j \in \mathbb{N}$, where $1<p<2$. Then, by (2.16) in Theorem 2.1, one obtains that the solution $\left(\varphi_{1}, \varphi_{2}\right)^{T}$ of $(2.15)$ satisfies

$$
\left\|\varphi_{1}\right\|_{L_{\alpha}^{p}(\Gamma)}+\left\|\varphi_{2}\right\|_{L_{\alpha}^{p}(\Gamma)} \leq C\left(\left\|f_{1 j}\right\|_{L_{\alpha}^{p}(\Gamma)}+\left\|f_{2 j}\right\|_{L_{\alpha}^{p}(\Gamma)}\right), \quad 1<p<2 .
$$

We next prove that the operator $S_{1 j}: L_{\alpha}^{p}(\Gamma) \rightarrow L_{\alpha}^{2}(\Gamma \backslash B)$ is uniformly bounded for $j \in \mathbb{N}$, where $1<p<2$. Indeed, by direct calculations, we can deduce that

$$
\begin{aligned}
& \left\|\int_{\Gamma} G_{1}\left(\cdot, y ; k_{1}\right) \varphi_{1}(y) d s(y)\right\|_{L^{2}(\Gamma \backslash B)} \\
& \quad=\sup _{\psi \in L_{\alpha}^{2},\|\psi\|_{L_{\alpha}^{2}(\Gamma \backslash B)}=1}\left|\int_{\Gamma \backslash B} \int_{\Gamma} G_{1}\left(x, y ; k_{1}\right) \varphi_{1}(y) d s(y) \psi(x) d x\right| \\
& \quad=\sup _{\psi \in L_{\alpha}^{2},\|\psi\|_{L_{\alpha}^{2}(\Gamma \backslash B)}=1}\left|\int_{\Gamma} \int_{\Gamma \backslash B} G_{1}\left(x, y ; k_{1}\right) \psi(x) d x \varphi_{1}(y) d s(y)\right| \\
& \quad \leq|\Gamma|^{\frac{1}{q}} \sup _{\psi \in L_{\alpha}^{2},\|\psi\|_{L_{\alpha}^{2}(\Gamma \backslash B)}=1} \sup _{y \in \Gamma \backslash B}\left\|G_{1}\left(\cdot, y ; k_{1}\right)\right\|_{L_{\alpha}^{2}(\Gamma \backslash B)}\|\psi\|_{L_{\alpha}^{2}(\Gamma \backslash B)}\left\|\varphi_{1}\right\|_{L_{\alpha}^{p}(\Gamma)} \\
& \quad=|\Gamma|^{\frac{1}{q}} \sup _{y \in \Gamma \backslash B}\left\|G_{1}\left(\cdot, y ; k_{1}\right)\right\|\left\|_{L_{\alpha}^{2}(\Gamma \backslash B)}\right\| \varphi_{1}\left\|_{L_{\alpha}^{p}(\Gamma)} \leq C\right\| \varphi_{1} \|_{L_{\alpha}^{p}(\Gamma)} .
\end{aligned}
$$

Here, we have used the fact that $G_{1}\left(\cdot, y ; k_{1}\right)$ is smooth on the boundary $\Gamma \backslash B$ in the first inequality. Then we have that $S_{1 j}: L_{\alpha}^{p}(\Gamma) \rightarrow L_{\alpha}^{2}(\Gamma \backslash B)$ is uniformly bounded for $j \in \mathbb{N}_{+}$. Moreover, by using similar arguments as those in the proof of (2.22), it is seen that the operators $S_{i j}, K_{i j}, K_{i j}^{\prime}$, and $T_{i j}$ are all uniformly bounded from $L_{\alpha}^{p}(\Gamma)$ into $L_{\alpha}^{2}(\Gamma \backslash B)$ for $j \in \mathbb{N}_{+}$, $i=1$, 2. Also notice that $f_{1}(j), f_{2}(j) \in L_{\alpha}^{2}(\Gamma \backslash B)$ are uniformly bounded for $j \in \mathbb{N}_{+}$. This, combined with equation (2.15), gives that the unique solution $\left(\varphi_{1}, \varphi_{2}\right)^{T}$ of $(2.15)$ satisfies that $\left(\varphi_{1}, \varphi_{2}\right)^{T} \in L_{\alpha}^{2}(\Gamma \backslash B) \times L_{\alpha}^{2}(\Gamma \backslash B)$. It is noted from (2.14) that the solution $u_{2 j}$ of the transmission problem (2.1)-(2.5) can be rewritten in the form of

$$
\begin{aligned}
u_{2 j}(x)= & \int_{\Gamma \backslash B} G_{2}\left(x, y ; k_{2}\right) \varphi_{1}(y) d s(y)+\int_{\Gamma \cap B} G_{2}\left(x, y ; k_{2}\right) \varphi_{1}(y) d s(y) \\
& +\int_{\Gamma \backslash B} \frac{\partial G_{2}\left(x, y ; k_{2}\right)}{\partial v(y)} \varphi_{2}(y) d s(y)+\int_{\Gamma \cap B} \frac{\partial G_{2}\left(x, y ; k_{2}\right)}{\partial v(y)} \varphi_{2}(y) d s(y) .
\end{aligned}
$$


Define

$$
S_{\Gamma \backslash B}(j) \varphi_{1}:=\int_{\Gamma \backslash B} G_{2}\left(x, y ; k_{2}\right) \varphi_{1}(y) d s(y) .
$$

It is easily seen that $S_{\Gamma \backslash B}(j): H_{\alpha}^{-\frac{1}{2}}(\Gamma \backslash B) \rightarrow H_{\alpha}^{\frac{1}{2}}(\Gamma \backslash B)$ is uniformly bounded for $j \in \mathbb{N}$. This in combination with the fact that $\varphi_{1} \in L_{\alpha}^{2}(\Gamma \backslash B)$ implies that $q_{1 j}(x):=S_{\Gamma \backslash B}(j) \varphi_{1}$ satisfies the following Dirichlet problem:

$$
\begin{cases}\triangle w+k_{2}^{2} w=0 & \text { in } \Omega_{-} \backslash B \\ w=q_{1 j} \in H_{\alpha}^{\frac{1}{2}}(\tilde{\Gamma}) & \text { on } \tilde{\Gamma}, \\ w(x)=\sum_{n \in \mathbb{Z}^{2}} w_{n}^{-} \exp \left(i \alpha_{n} \cdot \tilde{x}-i \beta_{n}^{-} x_{3}\right) & x_{3}<A_{2}\end{cases}
$$

where $\tilde{\Gamma}=(\Gamma \backslash B) \cup\left(\partial B \cap \Omega_{-}\right)$. Then the well-posedness of the Dirichlet problem (2.24) yields that, for any $h \in \mathbb{R}, q_{1 j} \in H^{1}\left(\Omega_{-}(h) \backslash \bar{B}\right)$ uniformly for $j \in \mathbb{N}_{+}$.

We now define

$$
q_{2 j}(x):=\int_{\Gamma \cap B} G_{2}\left(x, y ; k_{2}\right) \varphi_{1}(y) d s(y) .
$$

Since the region $\Omega_{-} \backslash B$ has a positive distance from $y_{0}$, it is found that $q_{2 j}(x) \in H^{1}\left(\Omega_{-}(h) \backslash\right.$ $\bar{B})$ uniformly for $j \in \mathbb{N}_{+}$. We further define

$$
K_{\Gamma \backslash B}(j) \varphi_{2}:=\int_{\Gamma \backslash B} \frac{\partial G_{2}\left(x, y ; k_{2}\right)}{\partial v(y)} \varphi_{2}(y) d s(y) .
$$

Obviously, $K_{\Gamma \backslash B}(j): H_{\alpha}^{-\frac{1}{2}}(\Gamma \backslash B) \rightarrow H_{\alpha}^{\frac{1}{2}}(\Gamma \backslash B)$ is uniformly bounded for $j \in \mathbb{N}_{+}$. Then, by the fact that $\varphi_{2} \in L_{\alpha}^{2}(\Gamma \backslash B)$, we obtain that $q_{3 j}(x):=K_{\Gamma \backslash B}(j) \varphi_{2}$ satisfies the Dirichlet problem (2.24), with the boundary data $w=q_{1 j}$ replaced by $w=q_{3 j}$ on $\tilde{\Gamma}$. Then using similar arguments as those in the proof of $q_{1 j} \in H_{\alpha}^{1}\left(\Omega_{-}(h) \backslash \bar{B}\right)$ yields that $q_{3 j} \in H_{\alpha}^{1}\left(\Omega_{-}(h) \backslash \bar{B}\right)$ uniformly for $j \in \mathbb{N}_{+}$. We also define

$$
q_{4 j}(x):=\int_{\Gamma \cap B} \frac{\partial G_{2}\left(x, y ; k_{2}\right)}{\partial v(y)} \varphi_{2}(y) d s(y) .
$$

The uniform boundedness of $q_{4 j} \in H_{\alpha}^{1}\left(\Omega_{-}(h) \backslash \bar{B}\right)$ for $j \in \mathbb{N}_{+}$can be concluded from the positive distance between the region $\left(\Omega_{-}(h) \backslash \bar{B}\right)$ and $y_{0}$. Finally, the desired result (2.20) follows from the discussions below (2.24). The proof of the theorem is thus completed.

\section{Uniqueness of the inverse problem}

In this section we mainly focus on the inverse problem of determining the periodic interface by means of the near-field data measured from one side of the periodic interface. To address this issue, we first introduce a mixed-reciprocity relation between the incident plane wave (1.1) and the incident point source (2.6). To accomplish this, we let $\hat{\alpha}:=-\alpha$ and consider an incident point source located at $z \in \Omega_{+}$taking the form

$$
G_{1}\left(x, z ; k_{1}\right)=\frac{i}{8 \pi^{2}} \sum_{n \in \mathbb{Z}^{2}} \frac{1}{\hat{\beta}_{n}^{+}} \exp \left(i \hat{\alpha}_{n} \cdot(\widetilde{x}-\widetilde{z})+i \hat{\beta}_{n}^{+}\left|x_{3}-z_{3}\right|\right), \quad x \neq z
$$


with the coefficients $\hat{\alpha}_{n}, \hat{\beta}_{n}^{+}$defined by $\alpha_{n}, \beta_{n}^{+}$with $\alpha$ replaced by $\hat{\alpha}$, respectively. Then the inverse scattering of the incident point source $G_{1}\left(\cdot, z ; k_{1}\right)$ by the two-layered periodic interface can be formulated as the following $\hat{\alpha}$-quasi-periodic problem:

$$
\begin{aligned}
& \triangle \hat{v}_{1}+k_{1}^{2} \hat{v}_{1}=0 \quad \text { in } \Omega_{+} \backslash\{z\}, \\
& \triangle \hat{v}_{2}+k_{2}^{2} \hat{v}_{2}=0 \quad \text { in } \Omega_{-}, \\
& \hat{v}_{1}=\hat{v}_{2}, \quad \frac{\partial \hat{v}_{1}}{\partial v}=\lambda \frac{\partial \hat{v}_{2}}{\partial v} \quad \text { on } \Gamma, \\
& \hat{v}^{s}(x)=\sum_{n \in \mathbb{Z}^{2}} \hat{v}_{n}^{+} \exp \left(i \hat{\alpha}_{n} \cdot \tilde{x}+i \hat{\beta}_{n}^{+} x_{3}\right), \quad x_{3}>A_{1}, \\
& \hat{v}_{2}(x)=\sum_{n \in \mathbb{Z}^{2}} \hat{v}_{n}^{-} \exp \left(i \hat{\alpha}_{n} \cdot \tilde{x}-i \hat{\beta}_{n}^{-} x_{3}\right), \quad x_{3}<A_{2} .
\end{aligned}
$$

Here, both $\hat{v}_{1}$ in $\Omega_{+}$and $\hat{v}_{2}$ in $\Omega_{-}$satisfy the $\hat{\alpha}$-quasi-periodic condition

$$
\hat{v}_{j}\left(\widetilde{x}+2 n \pi, x_{3}\right)=e^{i 2 \hat{\alpha} \cdot n \pi} \hat{v}_{j}\left(\widetilde{x}, x_{3}\right), \quad j=1,2 .
$$

Moreover, we write the scattered field $\hat{v}^{s}(\cdot, z):=\hat{v}_{1}(\cdot, z)-G_{1}\left(\cdot, z ; k_{1}\right)$ indicates the dependance of the wave field on the location of the point source, and let $v(\cdot ; m)$ and $u^{s}(\cdot ; m)$ be the scattered solution to problem (1.2)-(1.6) with respect to the incident wave $u^{i}(x ; m)=$ $\exp \left(i \alpha_{m} \cdot \tilde{x}-i \beta_{m}^{+} x_{3}\right), m \in \mathbb{Z}^{2}$. Therefore, we have the following mixed-reciprocity relation (for a proof, we refer to [34, Lemma 4.1]).

Lemma 3.1 For $z_{0} \in \Omega_{+}$, let $\hat{v}_{n}^{+}\left(z_{0}\right)$ be the Rayleigh coefficients of $\hat{v}_{1}^{s}\left(\cdot ; z_{0}\right)$. Then it holds that

$$
u_{1}^{s}\left(z_{0} ; m\right)=-8 \pi^{2} i \hat{\beta}_{-m}^{+} \hat{v}_{-m}^{+}\left(z_{0}\right) \text { for all } m \in \mathbb{Z}^{2} .
$$

Now we are in a position to present a uniqueness theorem for our inverse problem. The proof mainly depends on the a priori estimates established in Sect. 2 and a construction of a well-posed transmission problem in a sufficiently small domain.

Theorem 3.2 Let $u_{1}^{s}(\cdot ; m)$ and $\widetilde{u}_{1}^{s}(\cdot ; m)$ be the scattered fields corresponding to problem (1.2)-(1.6) with respect to the different bi-periodic interfaces $\Gamma$ and $\widetilde{\Gamma}$, respectively, induced by the same incident field $u^{i}(x ; m)=\exp \left(i \alpha_{m} \cdot \tilde{x}-i \beta_{m}^{+} x_{3}\right), m \in \mathbb{Z}^{2}$. If $\left.u_{1}^{s}(\cdot ; m)\right|_{\Gamma_{+}(h)}=$ $\left.\widetilde{u}_{1}^{s}(\cdot ; m)\right|_{\Gamma_{+}(h)}$ for all incident fields $u^{i}(x ; m) m \in \mathbb{Z}^{2}$, then we have $\Gamma=\widetilde{\Gamma}$.

Proof We shall prove the assertion by contradiction. Assume contrarily that $\Gamma \neq \widetilde{\Gamma}$. Without loss of generality, we can choose a point $z^{*} \in \Gamma \backslash \widetilde{\Gamma}$ satisfying that $f\left(\widetilde{z}^{*}\right)>\widetilde{f}\left(\widetilde{z}^{*}\right)$ with $z^{*}=\left(z^{*}, z_{3}\right)$. Then we define the sequence

$$
z_{j}:=z^{*}-\frac{\delta}{j} v\left(z^{*}\right) \quad \text { for } j=1,2, \ldots \cdots
$$

with sufficiently small $\delta>0$ such that $z_{j} \in B_{\varepsilon_{0}}\left(z^{*}\right) \subseteq\left(\Omega_{+} \cap \widetilde{\Omega}_{+}\right)$for all $j \in \mathbb{N}_{+}$, where $B_{\varepsilon_{0}}\left(z^{*}\right)$ is a small ball centered at $z^{*}$ with the radius $\varepsilon_{0}>0$. 
Let $\left(\hat{v}_{1}\left(\cdot ; z_{j}\right), \hat{v}_{2}\left(\cdot ; z_{j}\right)\right)$ and $\left(\hat{v}_{1}\left(\cdot ; z_{j}\right), \hat{v}_{2}\left(\cdot ; z_{j}\right)\right)$ be the solutions to problem $(3.2)-(3.6)$ corresponding to the same $\hat{\alpha}$-quasi-periodic incident point source $\hat{v}^{i}=\hat{G}\left(\cdot, z_{j}\right)$ with $z_{j}$ defined by (3.8). Then one obtains from Lemma 3.1 that

$$
u_{1}^{s}\left(z_{j} ; m\right)=-8 \pi^{2} i \hat{\beta}_{-m}^{+} \hat{v}_{-m}^{+}\left(z_{j}\right) \quad \text { and } \quad \widetilde{u}_{1}^{s}\left(z_{j} ; m\right)=-8 \pi^{2} i \hat{\beta}_{-m}^{+} \hat{\widetilde{v}}_{-m}^{+}\left(z_{j}\right)
$$

for all $m \in \mathbb{Z}^{2}$, where $\hat{v}_{-m}^{+}\left(z_{j}\right)$ and $\hat{v}_{-m}^{+}\left(z_{j}\right)$ denote the Rayleigh coefficients of the scattered fields $\hat{v}^{s}\left(\cdot ; z_{j}\right)$ and $\hat{\hat{v}}\left(\cdot ; z_{j}\right)$, respectively. By the assumption that $\left.u_{1}^{s}(\cdot ; m)\right|_{\Gamma_{+}(h)}=\left.\widetilde{u}_{1}^{s}(\cdot ; m)\right|_{\Gamma_{+}(h)}$ for all incident fields $u^{i}(x ; m) m \in \mathbb{Z}^{2}$, we arrive at that $\hat{v}_{-m}^{+}\left(z_{j}\right)=\hat{v}_{-m}^{+}\left(z_{j}\right), m \in \mathbb{Z}^{2}$. This in combination with the Rayleigh expansions and the unique continuation principle implies that

$$
\hat{v}_{1}\left(\cdot ; z_{j}\right)=\hat{\widetilde{v}}_{1}\left(\cdot ; z_{j}\right) \quad \text { in } \Omega^{+} \cap \widetilde{\Omega}^{+}
$$

for all $j \in \mathbb{N}_{+}$.

Denote $D_{0}:=B_{\varepsilon_{0}}\left(z^{*}\right) \cap \Omega^{-}$with sufficiently small $\varepsilon_{0}>0$ such that $D_{0} \subseteq\left(\Omega_{-} \cap \widetilde{\Omega}_{+}\right)$. Let $U_{j}:=\hat{v}_{1}\left(\cdot ; z_{j}\right)$ and $W_{j}:=\hat{v}_{2}\left(\cdot ; z_{j}\right)$, it is observed that $\left(U_{j}, W_{j}\right)$ satisfies the following modified interior transmission problem:

$$
\begin{cases}\Delta U_{j}-U_{j}=g_{1, j} & \text { in } D_{0}, \\ \Delta W_{j}-W_{j}=g_{2, j} & \text { in } D_{0}, \\ U_{j}-W_{j}=h_{1, j} & \text { on } \partial D_{0}, \\ \frac{\partial U_{j}}{\partial v}-\lambda \frac{\partial W_{j}}{\partial v}=h_{2, j} & \text { on } \partial D_{0}\end{cases}
$$

with the right terms and the boundary data

$$
\begin{array}{ll}
g_{1, j}:=-\left(k_{1}^{2}+1\right) \hat{\tilde{v}}_{1}\left(\cdot ; z_{j}\right), & g_{2, j}:=-\left(k_{2}^{2}+1\right) \hat{v}_{2}\left(\cdot ; z_{j}\right), \\
h_{1, j}:=\hat{\widetilde{v}}_{1}\left(\cdot ; z_{j}\right)-\hat{v}_{2}\left(\cdot ; z_{j}\right), \quad & h_{2, j}:=\frac{\partial \hat{\widetilde{v}}_{1}\left(\cdot ; z_{j}\right)}{\partial v}-\lambda \frac{\partial \hat{v}_{2}\left(\cdot ; z_{j}\right)}{\partial v} .
\end{array}
$$

Clearly, one has that $h_{1, j}=h_{2, j}$ on $\partial D_{0} \cap \Gamma$. Since $Z^{*}$ has a positive distance from $\widetilde{\Gamma}$, we obtain that $\hat{\widetilde{v}}\left(\cdot ; z_{j}\right) \in H^{1}\left(D_{0}\right)$ uniformly for all $j \in \mathbb{N}_{+}$. In view of the fact that $\hat{G}\left(\cdot, z_{j}\right) \in L^{2}\left(D_{0}\right)$ uniformly for all $j \in \mathbb{N}_{+}$, it is deduced that $g_{1, j} \in L^{2}\left(D_{0}\right)$ uniformly for all $j \in \mathbb{N}_{+}$. The uniform boundedness of $g_{2, j}$ in $L^{2}\left(D_{0}\right)$ for all $j \in \mathbb{N}_{+}$is a direct consequence of Corollary 2.2 in Sect. 2. Moreover, arguing similarly as in [36, Theorem 2.9], one derives from the fact that $h_{1, j}=h_{2, j}$ on $\partial D_{0} \cap \Gamma$ that $h_{1, j} \in H^{1 / 2}\left(\partial D_{0}\right)$ and $h_{2, j} \in H^{-1 / 2}\left(\partial D_{0}\right)$, respectively, uniformly for all $j \in \mathbb{N}_{+}$. Therefore, by the well-posedness of problem (3.11), we have

$$
\left\|\hat{G}\left(\cdot, z_{j}\right)\right\|_{H^{1}\left(D_{0}\right)}-\left\|\hat{\tilde{v}}\left(\cdot ; z_{j}\right)\right\|_{H^{1}\left(D_{0}\right)} \leq\left\|\hat{\widetilde{v}}\left(\cdot ; z_{j}\right)\right\|_{H^{1}\left(D_{0}\right)}=\left\|U_{j}\right\|_{H^{1}\left(D_{0}\right)} \leq C
$$

However, the above inequality is a contradiction since $\left\|\hat{\tilde{v}}^{s}\left(\cdot ; z_{j}\right)\right\|_{H^{1}\left(D_{0}\right)}$ is uniformly bounded and $\left\|\hat{G}\left(\cdot, z_{j}\right)\right\|_{H^{1}\left(D_{0}\right)} \rightarrow \infty$ as $j \rightarrow \infty$. Therefore, one concludes that $\Gamma=\widetilde{\Gamma}$. This completes the proof of the theorem. 


\section{Funding}

This work was supported by the NNSF of China under grants 11871416, 11971273 and by the projects ZR2019MA027, ZR2018MA004, and ZR2017MA044 supported by Shandong Provincial Natural Science Foundation.

\section{Availability of data and materials}

Not applicable.

\section{Competing interests}

The authors declare that they have no competing interests.

\section{Authors' contributions}

All authors contributed equally to the writing of this paper. All authors read and approved the final manuscript.

\section{Author details}

${ }^{1}$ School of Mathematics and Information Sciences, Yantai University, Yantai, Shandong 264005, P.R. China. ${ }^{2}$ School of Mathematics and Information Science, Shandong Technology and Business University, Yantai, 264005, P.R. China.

\section{Publisher's Note}

Springer Nature remains neutral with regard to jurisdictional claims in published maps and institutional affiliations.

Received: 24 September 2020 Accepted: 1 December 2020 Published online: 09 December 2020

\section{References}

1. Adams, A., Fournier, J.F.: Sobolev Spaces, 2nd edn. Elsevier, Singapore (2003)

2. Ammari, H.: Uniqueness theorems for an inverse problem in a doubly periodic structure. Inverse Probl. 11, 823-833 (1995)

3. Arens, T., Grinberg, N.: A complete factorization method for scattering by periodic surfaces. Computing 75, 111-132 (2005)

4. Arens, T., Kirsch, A.: The factorization method in inverse scattering from periodic structures. Inverse Probl. 19, 1195-1211 (2003)

5. Bao, G.: A uniqueness theorem for an inverse problem in periodic diffractive optics. Inverse Probl. 10, 335-340 (1994)

6. Bao, G., Zhang, H., Zou, J.: Unique determination of periodic polyhedral structures by scattered electromagnetic fields. Trans. Am. Math. Soc. 363, 4527-4551 (2011)

7. Bao, G., Zhou, Z:: An inverse problem for scattering by a doubly periodic structure. Trans. Am. Math. Soc. 350, 4089-4103 (1998)

8. Colton, D., Kress, R.: Integral Equation Methods in Scattering Theory. Wiley, New York (1983)

9. Colton, D., Kress, R., Monk, P.: Inverse scattering from an orthotropic medium. J. Comput. Appl. Math. 81, 269-298 (2007)

10. Elschner, J., Hsiao, G.C., Rathsfeld, A.: Grating profile reconstruction based on finite elements and optimization techniques. SIAM J. Appl. Math. 64, 525-545 (2003)

11. Elschner, J., Hu, G.: Global uniqueness in determining polygonal periodic structures with a minimal number of incident plane waves. Inverse Probl. 26, 115002 (2010)

12. Elschner, J., Schmidt, G., Yamamoto, M.: An inverse problem in periodic diffractive optics: global uniqueness with a single wave number. Inverse Probl. 19, 779-787 (2003)

13. Elschner, J., Yamamoto, M.: Uniqueness results for an inverse periodic transmission problem. Inverse Probl. 20 1841-1852 (2004)

14. Elschner, J., Yamamoto, M.: Uniqueness in determining polygonal periodic structures. Z. Anal. Anwend. 26, 165-177 (2007)

15. Gilbarg, D., Trudinger, N.S.: Elliptic Partial Differential Equations of Second Order, 2nd edn. Springer, New York (1983)

16. Haddar, H., Nguyen, T.P.: Sampling methods for reconstructing the geometry of a local perturbation in unknown periodic layers. Comput. Math. Appl. 74, 2831-2855 (2017)

17. Harris, I., Nguyen, D.L., Sands, J., Truong, T.: On the inverse scattering from anisotropic periodic layers and transmission eigenvalues. Appl. Anal. (2020). https://doi.org/10.1080/00036811.2020.1836349

18. Hettlich, F., Kirsch, A.: Schiffer's theorem in inverse scattering theory for periodic structures. Inverse Probl. 13, 351-361 (1997)

19. Hu, G., Qu, F., Zhang, B.: Direct and inverse problems for electromagnetic scattering by a doubly periodic structure with a partially coated dielectric. Math. Methods Appl. Sci. 33, 147-156 (2010)

20. Hu, G., Qu, F., Zhang, B.: A linear sampling method for inverse problems of diffraction gratings of mixed type. Math. Methods Appl. Sci. 35, 1047-1066 (2012)

21. Hu, G., Yang, J., Zhang, B.: An inverse electromagnetic scattering problem for a bi-periodic inhomogeneous layer on a perfectly conducting plate. Appl. Anal. 90, 317-333 (2011)

22. Hu, G., Zhang, B.: The linear sampling method for the inverse electromagnetic scattering by a partially coated bi-periodic structure. Math. Methods Appl. Sci. 34, 509-519 (2011)

23. Kirsch, A.: Uniqueness theorems in inverse scattering theory for periodic structures. Inverse Probl. 10, 145-152 (1994)

24. Kirsch, A.: An inverse problem for periodic structures. In: Kleinman, R.E., Kress, R., Martensen, E. (eds.) Inverse Scattering and Potential Problems Mathematical Physics pp. 75-93. Peter Lang, Frankfurt (1995)

25. Nguyen, T.P.: Differential imaging of local perturbations in anisotropic periodic media. Inverse Probl. 36, 034004 (2020)

26. Potthast, R:: On the convergence of a new Newton-type method in inverse scattering. Inverse Probl. 17, 1419-1434 (2001) 
27. Qu, F., Yang, J.: On recovery of an inhomogeneous cavity in inverse acoustic scattering. Inverse Probl. Imaging 12, 281-291 (2018)

28. Qu, F., Yang, J., Zhang, B.: Recovering an elastic obstacle containing embedded objects by the acoustic far-field measurements. Inverse Probl. 34, 015002 (2018)

29. Qu, F., Yang, J., Zhang, H.: Shape reconstruction in inverse scattering by an inhomogeneous cavity with internal measurements. SIAM J. Imaging Sci. 12, 788-808 (2019)

30. Qu, F., Zhang, B., Zhang, H.: A novel integral equation for scattering by locally rough surfaces and application to the inverse problem: the Neumann case. SIAM J. Sci. Comput. 41, A3673-A3702 (2019)

31. Strycharz, B.: An acoustic scattering problem for periodic, inhomogeneous media. Math. Methods Appl. Sci. 21, 969-983 (1998)

32. Strycharz, B.: Uniqueness in the inverse transmission scattering problem for periodic media. Math. Methods Appl. Sci. 22, 753-772 (1998)

33. Yang, J., Zhang, B.: An inverse transmission scattering problem for periodic media. Inverse Probl. 27, 125010 (2011)

34. Yang, J., Zhang, B.: Uniqueness results in the inverse scattering problem for periodic structures. Math. Methods Appl. Sci. 35, 828-838 (2012)

35. Yang, J., Zhang, B., Zhang, H.: A sampling method for the inverse transmission problem for periodic media. Inverse Probl. 28, 035004 (2012)

36. Yang, J., Zhang, B., Zhang, H.: Uniqueness in inverse acoustic and electromagnetic scattering by penetrable obstacles. J. Differ. Equ. 12, 6352-6383 (2018)

\section{Submit your manuscript to a SpringerOpen ${ }^{\circ}$ journal and benefit from:}

- Convenient online submission

- Rigorous peer review

- Open access: articles freely available online

- High visibility within the field

- Retaining the copyright to your article

Submit your next manuscript at $\gg$ springeropen.com 